\title{
Prevalence of methicillin-resistant Staphylococcus haemolyticus in companion animals: a cross-sectional study
}

Modestas Ruzauskas*, Rita Siugzdiniene, Irena Klimiene, Marius Virgailis, Raimundas Mockeliunas, Lina Vaskeviciute and Dainius Zienius

\begin{abstract}
Background: Among coagulase-negative staphylococci, Staphylococcus haemolyticus is the second most frequently isolated species from human blood cultures and has the highest level of antimicrobial resistance. This species has zoonotic character and is prevalent both in humans and animals. Recent studies have indicated that methicillin-resistant S. haemolyticus (MRSH) is one of the most frequent isolated Staphylococcus species among neonates in intensive care units. The aim of this study was to determine the presence of MRSH in different groups of companion animals and to characterize isolates according their antimicrobial resistance.
\end{abstract}

Methods: Samples $(n=754)$ were collected from healthy and diseased dogs and cats, female dogs in pure-breed kennels, healthy horses, and kennel owners. Classical microbiological tests along with molecular testing including PCR and $16 \mathrm{~S}$ rRNA sequencing were performed to identify MRSH. Clonality of the isolates was assessed by Pulsed Field Gel Electrophoresis using the Smal restriction enzyme. Antimicrobial susceptibility testing was performed using the broth micro-dilution method. Detection of genes encoding antimicrobial resistance was performed by PCR. Statistical analysis was performed using the R Project of Statistical Computing, "R 1.8.1" package.

Results: From a total of 754 samples tested, 12 MRSH isolates were obtained. No MRSH were found in horses and cats. Eleven isolates were obtained from dogs and one from a kennel owner. Ten of the dog isolates were detected in pure-breed kennels. The isolates demonstrated the same clonality only within separate kennels.

The most frequent resistances of MRSH isolates was demonstrated to benzylpenicillin (91.7\%), erythromycin (91.7\%), gentamicin (75.0\%), tetracycline (66.7\%), fluoroquinolones (41.7\%) and co-trimoxazole (41.7\%). One isolate was resistant to streptogramins. All isolates were susceptible to daptomycin, rifampin, linezolid and vancomycin. The clone isolated from the kennel owner and one of the dogs was resistant to beta-lactams, macrolides, gentamicin and tetracycline.

Conclusions: Pure-breed kennels keeping 6 or more females were determined to be a risk factor for the presence of MRSH strains. MRSH isolated from companion animals were frequently resistant to some classes of critically important antimicrobials, although they remain susceptible to antibiotics used exclusively in human medicine.

Keywords: Staphylococcus haemolyticus, Methicillin-resistance, Kennels, Antimicrobial resistance, Companion animals

\section{Background}

The ongoing change in the relationship between humans and companion animals is hallmarked by increasing intensive care provided to companion animals in veterinary medicine, resulting in growing numbers of high-risk animal patients [1]. Due to the zoonotic potential of

\footnotetext{
* Correspondence: ruzauskas@lva.It

Microbiology and Virology Institute, Lithuanian University of Health Sciences, Mickeviciaus g. 9, Kaunas LT44307, Lithuania
}

resistant bacteria and the close contact of pets with their owners, investigations on the presumptive transmission and infection routes of different bacterial species should be performed. The bacteria genus Staphylococcus is one of the most prevalent in animals and humans. Methicillinresistant staphylococci are recognized as one of the most important threats for human and animal health $[2,3]$. In spite of methicillin-resistant $S$. aureus (MRSA) being recognized as the most important Staphylococcus species in 
human patients, the other species also cause severe infections $[4,5]$. These species mostly include coagulasenegative staphylococci (CoNS) such as S. epidermidis, S. saprophyticus, and many other species within this group [6].

Among CoNS, Staphylococcus haemolyticus is the second most frequently isolated species from human blood cultures, and has the highest level of antimicrobial resistance [6-8]. This species may also cause septicemia, peritonitis, otitis, and urinary tract infections [6]. Recent studies reveled that methicillin-resistant $S$. haemolyticus (MRSH) was one of the most frequently isolated Staphylococcus species among neonates in intensive care units $[9,10]$. Data about the prevalence of this species in companion animals are still scarce. Research from the Netherlands has found 4 isolates of MRSH from 11 multidrug-resistant staphylococci isolated from dogs and cats [11]. In Denmark, an MRSH clone was isolated from horses, staff members, and environmental sites [12]. Another methicillin-resistant clone was obtained from cat wounds and animal cages in Norway [13].

In clinical laboratory practice, it is often uncommon to identify CoNS up to the species level for different reasons, such as high species variety, unclear taxonomic position, and unreliable identification results using biochemical tests $[6,14]$. Moreover, there are no special treatment strategies suggested for infections caused by different CoNS species. Therefore, there is a lack of data about the prevalence of certain CoNS species in both humans and animals. Such data are important, as different Staphylococcus species might have different pathogenicity mechanisms, pathogenesis, or peculiar transmission aspects. It is particularly important to identify methicillin-resistant CoNS species in companion animals for better understanding of microbiome and disease ecology, as well as for the selection of more appropriate control options in case they are needed. The aim of this study was to determine the presence of MRSH as a possible zoonotic agent in companion animals and to characterize the isolates according their antimicrobial resistance.

\section{Materials and methods}

\section{Animals and sampling}

From 2012 to 2014, samples were collected from 450 dogs, 50 cats, and 250 horses in Lithuania. The samples included diseased (300 dogs, 50 cats, and 50 horses) and healthy animals (200 horses and 50 dogs). Samples from the diseased pets (skin and wound infections, otitis, keratitis, infections of the respiratory and gastro-intestinal tracts) were taken directly at small animal clinics. Samples from the diseased horses (wounds, respiratory and gastrointestinal tract infections) were taken at stables or directly on pastures. Sampling of diseased animals was performed from the sites of infection, whereas nasal (30 from dogs and 150 from horses) and groin (20 from dogs and 50 from horses) samples were taken from healthy animals. One hundred vaginal samples were taken from adult female dogs suffering from reproductive organ infections or in cases of other reproductive disorders (infertility, partus praematurus, abortion) directly at kennels. Samples were collected by veterinary surgeons using sterile Amies media swabs (Liofilchem, Italy) or other necessary instruments under aseptic conditions from the affected organs or presumptive places of infection. Samples were delivered to the laboratory within the same day. Anamneses data were analysed in case of MRSH detection. Four owners of the kennels voluntarily presented their own nasal samples using sterile cotton swabs. Ethics approval was obtained from Bioethics Centre of Lithuanian University of Health Sciences. Informed consent was obtained from each volunteer.

\section{Bacteriological analysis}

Clinical material was inoculated onto 5\% Sheep Blood Agar, Mannitol Salt Agar (Liofilchem, Italy) supplemented with $4 \mathrm{mg} / \mathrm{L}$ cefoxitin (Sigma-Aldrich) and Brilliance MRSA 2 Agar (Oxoid, Thermo Fisher, UK). Presumptive identification of Staphyloccus genus was based on the growth and morphology characteristics, catalase production, gram-staining and susceptibility to furazolidone. Species identification was performed only for the isolates that grew on Mannitol Salt Agar supplemented with $4 \mathrm{mg} / \mathrm{L}$ cefoxitin and/or and Brilliance MRSA 2 Agar. Single colonies were taken from the agar surface and re-cultivated on the Mannitol Salt Agar supplemented with cefoxitin and Brilliance MRSA 2 Agar with the aim to obtain pure cultures. Presumptive species identification was based on pigment and coagulase production, presence of protein $\mathrm{A}$ and clumping factor as well as on biochemical properties detected by using RapID Staph Plus (Thermo Scientific) identification system. In uncertain identification cases Matrix-Assisted Laser Desorption/Ionization Time-of-Flight (MALDI-TOF) analysis (VITEK ${ }^{\circ}$ MS, Biomérieux, France) was used as described previously [15].

\section{Molecular testing}

DNA material for molecular testing was obtained after bacterial lysis according to the extraction protocol prepared by the Community Reference Laboratory for Antimicrobial Resistance with slight modifications [16]. Briefly, a loopful of colonies were taken from the surface of Mueller Hinton Agar and transferred to phosphate buffered saline ( $\mathrm{pH}$ 7.3). The content was centrifuged for $5 \mathrm{~min}$. Then the supernatant was discarded and the pellet was re-suspended in Tris-EDTA (TE) buffer. The suspension was heated using a thermomixer at $100^{\circ} \mathrm{C}$ degrees for 10 minutes. Boiled suspension was transferred directly on ice and diluted by 1:10 in TE. 
Genus specific 16S rRNA gene was investigated by PCR using Staphylococcus genus specific oligonucleotide primers (Table 1). MRSH confirmation was obtained through detection of the mecA, mecC and S. haemolyticus speciesspecific nuc gene (Table 1). Species verification additionally was performed using $16 \mathrm{~S}$ rRNA sequencing. The universal primers $27 \mathrm{~F}$ and 515R were used as described previously [17]. Sequences were analysed using Molecular Evolutionary Genetic Analysis software (MEGA, version 6). Basic local alignment search tool (BLAST) was used for comparison of obtained sequences with sequences presented in the database of National Centre of Biotechnology Information.

Clonality of the isolates was assessed by Pulsed Field Gel Electrophoresis (PFGE) with SmaI restriction enzyme according to protocol decribed previously [26]. The definition of a PFGE cluster was based on a similarity cutt-off value of $80 \%$ using the unweighted pair group method (UPGMA).

\section{Antimicrobial susceptibility testing}

Antimicrobial susceptibility testing was performed using the broth micro-dilution method. Sensititre ${ }^{\bullet}$ plates and the ARIS 2X automated system (Thermo Scientific) were used with the following antimicrobials: oxacillin, benzylpenicillin, clindamycin, erythromycin, gentamicin, tetracycline, daptomycin, ciprofloxacin, levofloxacin, linezolid, quinupristin/dalfopristin, vancomycin, co-trimoxazole and rifampin. Interpretation of results was carried-out using manufacturers software $\left(\mathrm{SWIN}^{\circ}\right)$ adapted to clinical breakpoints of European Committee on antimicrobial susceptibility testing (EUCAST). As there is no clinical breakpoint set for benzylpenicillin for CoNS, the value of $0.12 \mathrm{mg} / \mathrm{L}$ designed for S. aureus was used. The quality control strain

Table 1 Oligonucleotide primers used in this study

\begin{tabular}{|c|c|c|c|c|}
\hline Primer name & Sequence $\left(5^{\prime}-3^{\prime}\right)$ & Size, bp and $\mathrm{T}\left({ }^{\circ} \mathrm{C}\right)$ & Target gene & Source \\
\hline mecA1 & GGGATCATAGCGTCATTATTC & $527(61)$ & mecA & {$[16]$} \\
\hline mecA2 & AACGATTGTGACACGATAGCC & & & \\
\hline mecC1 & GCTCCTAATGCTAATGCA & $204(50)$ & mecC & [18] \\
\hline $\operatorname{mec} C 2$ & TAAGCAATAATGACTACC & & & \\
\hline $16 S 1$ & GTGCCAGCAGCCGCGGTAA & $886(61)$ & 165 staph & [16] \\
\hline 1652 & AGACCCGGGAACGTATTCAC & & & \\
\hline hae1 & TAGTGGTAGGCGTATTAGCC & $434(58)$ & nuc S. haemolyticus & [19] \\
\hline hae2 & ACGATATTTGCCATTCGGTG & & & \\
\hline blaZ1 & CAGTTCACATGCCAAAGAG & $772(50)$ & blaz & [20] \\
\hline blaZ2 & TACACTCTTGGCGGTTTC & & & \\
\hline tetM1 & GTTAAATAGTGTTCTTGGAG & $656(45)$ & tet(M) & [21] \\
\hline tetM2 & CTAAGATATGGCTCTAACAA & & & \\
\hline tetK1 & TTAGGTGAAGGGTTAGGTCC & $718(55)$ & $\operatorname{tet}(\mathrm{K})$ & [21] \\
\hline tetK2 & GCAAACTCATTCCAGAAGCA & & & \\
\hline aac6F & CAGAGCCTTGGGAAGATGAAG & $348(61)$ & $a a c\left(6^{\prime}\right)-1 e-a p h\left(2^{\prime \prime}\right)-1 a$ & [22] \\
\hline aac6R & CCTCGTGTAATTCATGTTCTGGC & & & \\
\hline aph3F & CCGCTGCGTAAAAGATAC & $609(57)$ & $a p h\left(3^{\prime}\right)-111 a$ & [22] \\
\hline aph3R & GTCATACCACTTGTCCGC & & & \\
\hline dftrG1 & TTCTITGATTGCTGCGATG & $501(51)$ & $d f r(G)$ & [23] \\
\hline $\mathrm{dfrG} 2$ & AACGCACCCGTTAACTCAAT & & & \\
\hline dfrk1 & GCTGCGATGGATAAGAACAG & $214(50)$ & $d f r(K)$ & [24] \\
\hline dfrk2 & GGACGATTTCACAACCATTAAAGC & & & \\
\hline ermA1 & AAGCGGTAAACCCCTCTGAG & $442(53)$ & $\operatorname{erm}(A)$ & {$[25]$} \\
\hline ermA2 & TCAAAGCCTGTCGGAATTGG & & & \\
\hline ermC1 & ATCTITGAAATCGGCTCAGG & $295(48)$ & $\operatorname{erm}(C)$ & [25] \\
\hline ermC2 & CAAACCCGTATTCCACGATT & & & \\
\hline msra1 & GCTTAACATGGATGTGG & $1230(55)$ & $m s r(A)$ & [22] \\
\hline msra2 & GATTGTCCTGTTAATTCCC & & & \\
\hline
\end{tabular}


S. aureus ATCC 29213 was included in each assay for validation purposes.

\section{PCR for detection of genes encoding antimicrobial resistance}

Detection of genes encoding antimicrobial resistance (mecA, mecC, blaZ, tet(K), tet $(\mathrm{M}), \operatorname{erm}(\mathrm{A}), \operatorname{erm}(\mathrm{C}), m s r$ (A), $\operatorname{aac}\left(6^{\prime}\right)-I e-\operatorname{aph}\left(2^{\prime \prime}\right)-I a, \operatorname{aph}\left(3^{\prime}\right)-I I I a, d f r(\mathrm{G})$ and $d f r(\mathrm{~K})$ was performed by PCR. Annealing temperatures and oligonucleotides used are presented in Table 1 .

\section{Statistical analysis}

Statistical analysis was performed using "R 1.8.1" package (http://www.r-project.org/). Comparison between categorical variables was calculated by chi-square and Fisher's exact test. Results were considered statistically significant if $\mathrm{P}<0.05$.

\section{Results}

From a total of 754 samples tested, $12 \mathrm{MRSH}$ isolates were obtained (1.6\%). No MRSH was found in horses or cats. Eleven isolates were obtained from dogs and one from the dog owner. Ten of the dog isolates were detected in kennels of pure-breed female dogs. Only one MRSH isolate was found in a non-pure-breed 6-year-old dog (male) suffering from deep pyoderma. There were no strains isolated from nose and groin in healthy animals. Three isolates were obtained from the same kennel (breeding English Bulldogs and French Bulldogs). These isolates (D1, D2, and D3) demonstrated the same clonality (Figure 1). They had similar antimicrobial characteristics as well.

One MRSH strain (H1) was obtained from the owner of another kennel (Yorkshire Terriers) and had similar clonality characteristics with one isolate (D11) obtained in this kennel. Two isolates (D4 and D5) from another kennel (breeding English Bulldogs) demonstrated clonality as well (similarity 96\%) and had restriction patterns that were close to those of the clone obtained in the first kennel breeding English Bulldogs and French Bulldogs (Figure 1). Those two clones had close antimicrobial susceptibility profiles as well. Small differences were detected in the biochemical properties (reduction of nitrates) among those two clones.

Analysis of the pedigree where two similar MRSH clones were found revealed the existence of the same paternal grandfather among two tested dogs. No other close relations have been registered among those two kennels. The fourth different MRSH clone (isolates D8 and D9, similarity 96\%) was detected among two isolates from a kennel breeding German Shepherds.

Statistically reliable results $(\mathrm{P}<0.05)$ for the presence of MRSH were obtained only in the cases of kennels with 6 or more breeding females. Anamnesis data revealed the usage of fluoroquinolones (enrofloxacin) in one MRSHpositive kennel, as well as fluoroquinolones and cephalosporins (cefovecin) in the other kennel during the last three months before sampling. The numbers of female dogs within those kennels were 6 and 18, respectively. The usage of antimicrobials was associated with reproductive disorders in female dogs within those kennels. All $\mathrm{MRSH}$ isolates harbored the $m e c A$ gene, while the $m e c C$ gene was not detected. Antimicrobial resistance patterns and resistance genes identified in MRSH strains are presented in Table 2.

The most frequent resistances of MRSH isolates demonstrated were to benzylpenicillin (91.7\%) with the presence of the blaZ gene; erythromycin (91.7\%) and clindamycin (41.7\%) with the presence of ermA, erm $C$, and $m s r A$ genes; and gentamicin (75.0\%) with the presence of $a a c$ (6')-Ie-aph(2")-Ia and aph(3')-IIIa genes. Resistance to fluoroquinolones was frequent: $41.7 \%$ of the isolates were resistant to ciprofloxacin. tet $K$ and/or tet $M$ genes were detected in eight tetracycline-resistant isolates (66.7\%). Two genes ( $d f r G$ or $d f r K$ ) were detected in co-trimoxazoleresistant (41.7\%) isolates (Table 1). All isolates were susceptible to daptomycin, rifampin, linezolid, and vancomycin. One isolate was resistant to streptogramins. The clone

\section{Isolates}

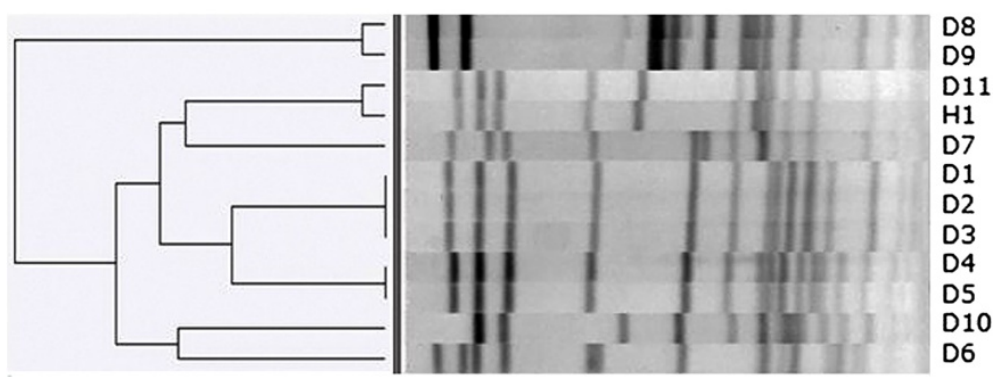

Figure 1 PFGE dendogram of genomic DNA of MRSH isolates. 
Table 2 Antimicrobial resistance patterns and resistance genes identified in MRSH strains

\begin{tabular}{|c|c|c|}
\hline Isolates & Resistance patterns & Resistance genes \\
\hline $\bar{D} 1^{a}, D 2^{a}, D 3^{a}$ & OX, P, E, CN, CIP, L, TE, SXT & mecA, blaZ, tetK, ermC, msrA, aac(6')-le-aph(2")-la, aph(3')-IIla, dfrG \\
\hline $\mathrm{D} 4^{\mathrm{c}}, \mathrm{D} 5^{\mathrm{c}}$ & $O X, P, E, C N, C I P, L, T E$ & mecA, blaZ, ermA, aac( $\left.6^{\prime}\right)$-le-aph $\left(2^{\prime \prime}\right)-1 a$, tetK \\
\hline D6 & $O X, P, E, Q / D$ & mecA, blaz \\
\hline D7 & OX, CN, TE & mecA, tetK, aac(6')-le-aph(2")-la, aph(3')-IIIa \\
\hline$D 8^{d}, D 9^{d}$ & OX, P, E, CLI, CN, SXT & 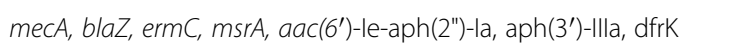 \\
\hline D10 & $\mathrm{OX}, \mathrm{P}, \mathrm{E}, \mathrm{CLI}$ & mecA, blaz, msrA \\
\hline $\mathrm{D} 11^{\mathrm{b}} \mathrm{H} 1^{\mathrm{b}}$, & OX, P, E, CLI, CN, TE & mecA, blaZ, msrA, tetM, aph(3')-IIIa \\
\hline
\end{tabular}

$\overline{a, b, c, d}$ - Isolates as the same clone with similar resistance profile; $\mathrm{D}$ - dog isolates; $\mathrm{H}$ - human isolate.

OX, oxacillin; P, benzylpenicillin; E, erythromycin; CLI, clindamycin; CN, gentamicin; CIP ciprofloxacin; L, levofloxacin; TE, tetracycline; Q/D, quinupristin/dalfopristin; SXT, co-trimoxazole.

isolated from a kennel owner and a dog was resistant to beta-lactams, macrolides, gentamicin, and tetracycline. The minimal inhibitory concentrations (MICs) of antimicrobials tested are presented in Figure 2. Statistically reliable correlation between MICs and detected genes was determined for tetracycline (tet $\mathrm{K}$ gene was associated with MIC16 mg/L), clindamycin ( $m s r \mathrm{~A}$ gene $2-4 \mathrm{mg} / \mathrm{L})$, gentamicin (aph(3')-IIIa and $a a c\left(6^{\prime}\right)$-Ie-aph $\left(2^{\prime \prime}\right)$-Ia genes $-\geq 4 \mathrm{mg} / \mathrm{L}$ ), erythromycin (ermC gene $4 \mathrm{mg} / \mathrm{L})$ and trimethoprim $(d f r G$ gene $-8 \mathrm{mg} / \mathrm{L})$.

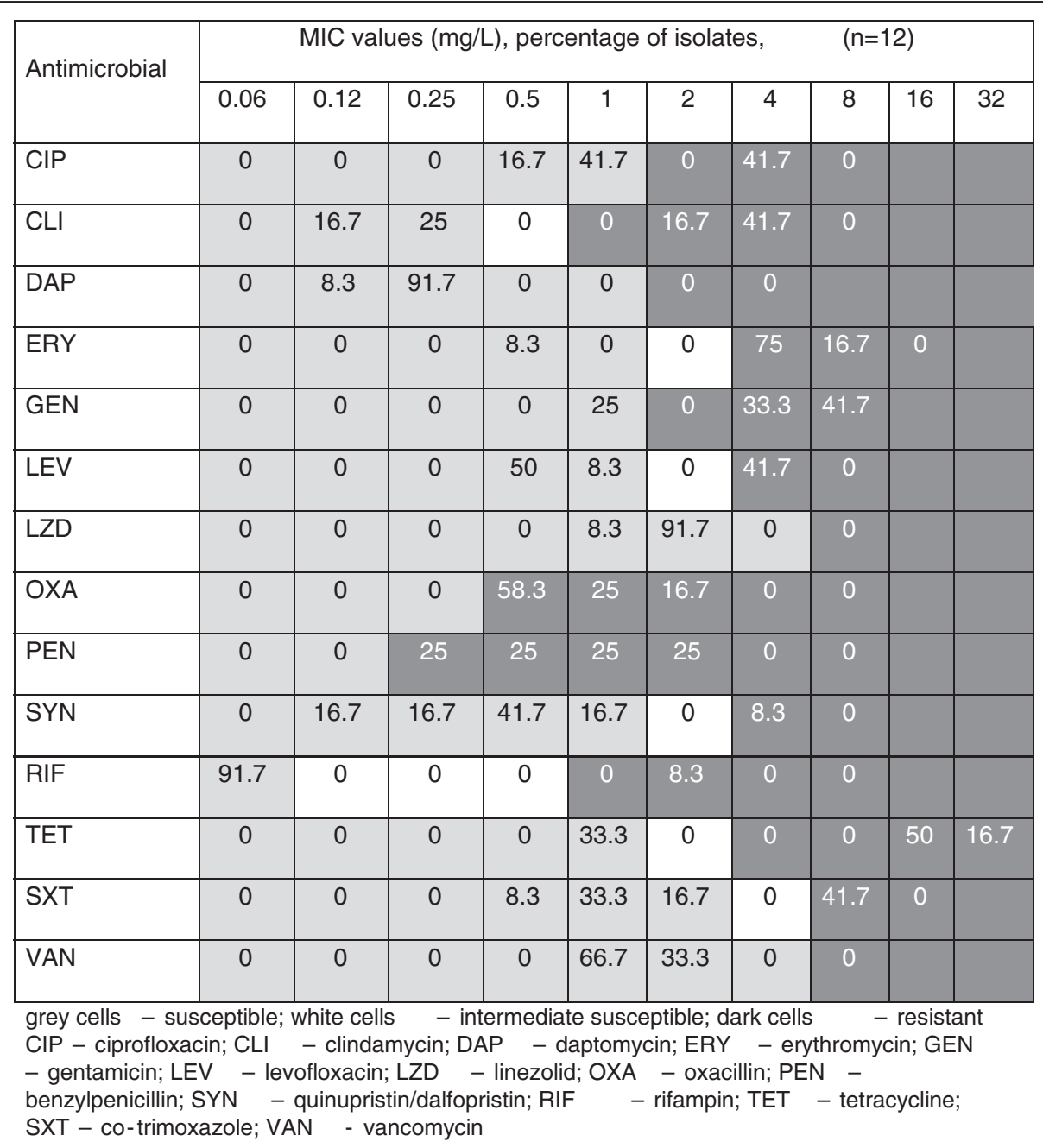

Figure 2 Minimum inhibitory concentration distributions for the MRSH isolates. 


\section{Discussion}

This study revealed that MRSH is prevalent in the population of dogs, and the occurrence of this species might be associated with specific distribution among certain animal groups. Ten out of twelve MRSH isolates have been detected in female dogs with reproductive disorders in kennels keeping pure-breed dogs. Previous studies did not reveal any specific distribution of this methicillinresistant species among different animal groups, although separate clones were detected in close-associated sources mostly animals, the environment, and equipment $[12,13]$. Reproductive organs of dogs were the main site of MRSH location, whereas no isolates have been found in nasal and groin samples. That might be associated with previous treatment of reproductive infections in bitches, i.e. antimicrobial selective pressure. There are no data about specific anatomical location of S. haemolyticus in dogs, although in humans it mostly colonize areas of axillae, perineus and groin [27].

We found MRSH clonality associated with a narrow distribution within separate kennels. The zoonotic character of $S$. haemolyticus is known based on previous facts according its distribution among humans, animals and ready-to-eat meat products [12,28]. Our findings support this, particularly by the fact that the same strain has been found in both dog and dog owner. As a rule, MRSH was detected in large kennels keeping no less than 6 adult female dogs. The data obtained might be important from an epidemiological point of view, as this study revealed a possible risk factor associated with animal concentration and MRS occurrence within the kennels. In spite of the fact that $S$. haemolyticus has previously been found in different animal species in other countries, including horses and cats, we did not find MRSH isolates in either in horses or in cats. This could be associated with the low frequency of methicillin-resistant Staphylococcus spp. among animals in Lithuania, as in our previous study, where we found only 4 MRS isolates in 520 livestock samples with no MRS occurrence in horses [29].

MRSH isolates demonstrated resistance to the antimicrobial classes recognized as critically important for humans - fluoroquinolones, macrolides, and aminoglycosides. Previously, intense usage of fluoroquinolones was reported for domestic animal treatment in Lithuania $[30,31]$. It was also proven that poultry products are frequently contaminated with fluoroquinolone-resistant bacteria $[31,32]$. Studies in other countries demonstrated frequent acquired resistance of MRSH to fluoroquinolones isolated from humans as well $[10,14]$.

Different genes encoding resistance to antimicrobials have been detected, which supports the opinion that this species could be characterized as highly resistant to antibiotics. The isolates remain susceptible only to vancomycin, linezolid, rifampin, and daptomycin - antibiotics that are not used for animal treatment.

\section{Conclusions}

Dogs were identified as the sole source of MRSH among companion animals in Lithuania. Pure-breed kennels keeping 6 or more females could be recognized as a risk factor for the presence of MRSH strains. MRSH carriers are a potential source for the possible transmission of MRS for their offspring, other animals, and humans in contact. MRSH strains isolated from companion animals were frequently resistant to some classes of critically important antimicrobials, although they remain susceptible to antibiotics exclusively used in human medicine.

\section{Abbreviations}

CoNS: Coagulase-negative staphylococci; MALDI-TOF: Matrix-assisted laser desorption/ionization time-of-flight analysis; MRS: Methicillin-resistant Staphylococcus; MRSH: Methicillin-resistant Staphylococcus haemolyticus; PCR: Polymerase chain reaction; PFGE: Pulse filed gel electrophoresis.

\section{Competing interests}

The authors declare that they have no competing interests.

\section{Authors' contributions}

MR and RM designed the sampling protocol and selected methods, IK and MV performed bacteriological a, LV and RS performed molecular testing, DZ performed statistical analysis, MR conceived the study and drafted the manuscript. All authors contributed and approved the submitted manuscript.

\section{Acknowledgements}

The authors thank Constança Pomba and Natacha Couto for consultancy and for assistance with molecular analysis. This research was funded by a grant (MIP-061/2012) from the Research Council of Lithuania.

Received: 30 August 2014 Accepted: 19 November 2014

Published online: 28 November 2014

\section{References}

1. Wieler LH, Ewers C, Guenther S, Walther B, Lübke-Becker A: Methicillin-resistant staphylococci (MRS) and extended-spectrum beta-lactamases (ESBL)-producing Enterobacteriaceae in companion animals: nosocomial infections as one reason for the rising prevalence of these potential zoonotic pathogens in clinical samples. Int J Med Microbiol 2011, 301:635-641.

2. Kluytmans JA: Methicillin-resistant Staphylococcus aureus in food products: cause for concern of case for complacency? Clin Microbiol Infect 2010, 16:11-15.

3. Catry B, Van Duijkeren E, Pomba MC, Greko C, Moreno MA, Pyörälä S, Ruzauskas M, Sanders P, Threlfall E, Ungemach F, Törneke K, Munoz-Madeiro C, Torren-Edo J: Reflection paper on MRSA in food-producing and companion animals: epidemiology and control options for human and animal health. Epidemiol Infect 2010, 138:626-644.

4. Chu VH, Woods CW, Miro JM, Hoen B, Cabell CH, Pappas PA, Federspiel J, Athan E, Stryjewski ME, Nacinovich F, Marco F, Levine DP, Elliott TS, Fortes CQ, Tornos P, Gordon DL, Utili R, Delahaye F, Corey GR, Fowler VG Jr: Emergence of coagulase-negative staphylococci as a cause of native valve endocarditis. Clin Infect Dis 2008, 46:232.

5. Rogers KL, Fey PD, Rupp ME: Coagulase-negative staphylococcal infections. Infect Dis Clin North Am 2009, 23:73-98.

6. Takeuchi F, Watanabe S, Baba T, Yuzawa H, Ito T, Morimoto Y, Kuroda M, Cui L, Takahashi M, Ankai A, Baba S, Fukui S, Lee JC, Hiramatsu K: Whole-genome sequencing ofStaphylococcus haemolyticus uncovers the extreme plasticity of its genome and the evolution of human-colonizing staphylococcal species. J Bacteriol 2005, 187:7292-7308. 
7. Barros EM, Ceotto H, Bastos MCF, DosSantos KRN, Giambiagi-deMarval M: Staphylococcus haemolyticus as an important hospital pathogen and carrier of methicillin resistance genes. J Clin Microbiol 2012, 50:166-168.

8. Froggatt JW, Johnston JL, Galetto DW, Archer GL: Antimicrobial resistance in nosocomial isolates of Staphylococcus haemolyticus. Antimicrob Agents Chemother 1989, 33:460-466.

9. Pereira PMA, Binatti VB, Sued BPR, Ramos JN, Peixoto RS, Simões C, DeCastro EA, Duarte JLMB, Vieira W, Hirata R Jr, Santos KRN, Guaraldi ALM, Pereira JAA: Staphylococcus haemolyticus dissemination among neonates with bacteremia in a neonatal intensive care unit Rio de Janeiro, Brasil. Diagn Microbiol Infect Dis 2014, 78:85-92.

10. Brzychczy M, Borszewska-Kornacka M, Gulczynska E, Wojkowska-Mach J, Sulik M, Grzebyk M, Luchter M, Heczko PB, Bulanda M: Prevalence of antibiotic resistance in multi-drug resistant coagulase-negative staphylococci isolated from invasive infection in very low birth weight neonates in two Polish NICUs. Ann Clin Microbiol Antimicrob 2013, 12:41.

11. VanDuijkeren E, Box ATA, Heck MEOC, Wannet WJB, Fluit AC: Methicillin-resistant staphylococci isolated from animals. Vet Microbiol 2004, 103:91-97.

12. Moodley A, Guardabassi L: Clonal spread of methicillin-resistant coagulase-negative staphylococci among horses, personnel and environmental sites at equine facilities. Vet Microbiol 2009, 137:397-401.

13. Sidhu MS, Oppegaard H, Devor TP, Sørum H: Persistence of multidrug-resistant Staphylococcus haemolyticus in an animal veterinary teaching hospital clinic. Microb Drug Resist 2007, 13:271-280.

14. Shittu A, Lin J, Morrison D, Kolawole D: Isolation and molecular characterization of multiresistant Staphylococcus sciuri and Staphylococcus haemolyticus associated with skin and soft-tissue infections. J Med Microbiol 2004, 53:51-55.

15. Dubois D, Grare M, Prere MF, Segonds C, Marty N, Oswald E: Performances of the Vitek MS matrix-assisted laser desorption ionization-time of flight mass spectrometry system for rapid identification of bacteria in routine clinical microbiology. J Clin Microbiol 2012, 50:2568-2576.

16. Anonymous: Multiplex PCR for the detection of the mecA gene and the identification of Staphylococcus aureus. In: National Food Institute (DTU) Protocol. National Food Institute, Technical University of Denmark, Copenhagen, 06.02.2008

17. Kim JH, Lee JY, Kim HR, Heo KW, Park SK, Lee JN, Yu SM, Shin JH: Acute lymphadenitis with cellulitis caused by Staphylococcus lugdunensis. Korean Lab J Med 2008, 28:196-200.

18. Cuny C, Layer F, Strommenger B, Witte W: Rare occurrence of methicillin-resistant Staphylococcus aureus CC130 with a novel mecA homologue in humans in Germany. PloS One 2011, 6(9):e24360.

19. Hirotaki S, Sasaki T, Kuwahara-Arai K, Hiramatsu K: Rapid and accurate identification of human-associated staphylococci by use of multiplex PCR. J Clin Microbiol 2011, 49:3627-3631.

20. Schnellmann C, Gerber V, Rossano A, Jaquier V, Panchaud Y, Doherr MG, Thomann A, Straub R, Perreten V: Presence of new mecA and mph (C) variants conferring antibiotic resistance in Staphylococcus spp. isolated from the skin of horses before and after clinic admission. J Clin Microbiol 2006, 44:4444-4454.

21. Aarestrup FM, Agerso $Y$, Gerner-Smidt P, Madsen M, Jensen LB: Comparison of antimicrobial resistance phenotypes and resistance genes in Enterococcus faecalis and Enterococcus faecium from humans in the community, broilers, and pigs in Denmark. Diagn Microbiol Infect Dis 2000, 37:127-137.

22. Perreten $V$, Vorlet-Fawer $L$, Slickers $P$, Ehricht $R$, Kuhnert $P$, Frey $J$ : Microarray-based detection of 90 antibiotic resistance genes of gram-positive bacteria. J Clin Microbiol 2005, 43:2291-2302.

23. Couto N, Belas A, Couto I, Perreten V, Pomba C: Genetic relatedness, antimicrobial and biocide susceptibility comparative analysis of methicillin-resistant and -susceptible Staphylococcus pseudintermedius from Portugal. Microb Drug Resist 2014, 20:364-371.

24. Kadlec K, Schwarz S: Identification of the novel dfrK-carrying transposon Tn559 in a porcine methicillin-susceptible Staphylococcus aureus ST398 strain. Antimicrob Agents Chemother 2010, 54:3475-3477.

25. Jensen LB, Hammerum AM, Bager F, Aarestrup FM: Streptogramin resistance among Enterococcus faecium isolated from production animals in Denmark in 1997. Microb Drug Resist 2002, 8:369-374.

26. Nunes APF, Teixeira LM, Bastos CCR, Silva MG, Ferreira RB, Fonseca LS, Santos KRN: Genomic characterization of oxacillin-resistant
Staphylococcus epidermidis and Staphylococcus haemolyticus isolated from Brazilian medical centers. J Hosp Infect 2005, 59:19-26.

27. Tristan A, Lina G, Etienne J, Vandenesch F: Biology and pathogenicity of staphylococci other than Staphylococcus aureus and Staphylococcus epidermidis. In Gram-positive Pathogens. Edited by Fischetti A, Novick RP, Ferretti JJ, Portnoy DA, Rood Jl. Washington: ASM Press; 2006:572-586.

28. Podkowik M, Bystroń J, Bania J: Prevalence of antibiotic resistance genes in staphylococci isolated from ready-to-eat meat products. Pol J Vet Sci 2012, 15:233-237.

29. Ruzauskas M, Couto N, Siugzdiniene R, Belas A, Klimiene I, Virgailis M, Pomba C: Occurence and characterization of livestock-associated methicillin-resistant Staphylococcus aureus. Vet Med Zoot 2014, 66:58-63.

30. Seputiene V, Povilonis J, Ruzauskas M, Virgailis M, Žlabys P, Suziedeliene E: Quinolone resistance among Salmonella enterica and Escherichia coli in Lithuania. Biologija 2006, 3:74-78.

31. Ruzauskas M, Siugzdiniene R, Seputiene V, Suziedeliene E, Virgailis M, Daugelavicius R, Spakauskas V, Zienius D, Sengaut J, Pavilonis A: The situation of antimicrobial resistance of enteric bacteria isolated from animal origin to quinolones and fluoroquinolones. Vet Med Zoot 2010, 50:73-80.

32. Ruzauskas M, Siugzdiniene R, Suziedeliene E, Seputiene V, Pavilonis J: Antimicrobial resistance of Enterococcus spp. spread in poultry products in Lithuania. J Food Saf 2010, 30:902-915.

doi:10.1186/s12941-014-0056-y

Cite this article as: Ruzauskas et al:: Prevalence of methicillin-resistant Staphylococcus haemolyticus in companion animals: a cross-sectional study. Annals of Clinical Microbiology and Antimicrobials 2014 13:56.

\section{Submit your next manuscript to BioMed Central and take full advantage of:}

- Convenient online submission

- Thorough peer review

- No space constraints or color figure charges

- Immediate publication on acceptance

- Inclusion in PubMed, CAS, Scopus and Google Scholar

- Research which is freely available for redistribution 